\title{
To Investigate the Mechanism of Hypertension Induced by Chronic Renal Failure-Related Arteriosclerosis and Principles of Treatment and Nursing Care in Traditional Chinese Medicine
}

\section{Chuang YC*}

Kiang Wu Nursing College of Macau, Macau SAR, China

*Corresponding author: Yao-Chen Chuang, Kiang Wu Nursing College of Macau, Est. Repouso No.35, R/C, Macau SAR. China, Tel: +853-85906389; Email: jasonchuang@kwnc.edu.mo

\section{Review Article \\ Volume 3 Issue 2}

Received Date: April 08, 2019

Published Date: April 17, 2019

DOI: $10.23880 /$ nhij-16000186

\section{Abstract}

Kidney disease is one of the causes of secondary hypertension; there are many mechanisms of hypertension, but the most basic one is arteriosclerosis. Recent studies have indicated a relationship between arteriosclerosis and the formation of advanced glycation end products (AGEs), arterial deposition, and difficulty in removing obstructions. Oxidative stress is one of the causes of AGEs formation. Studies have shown that chronic renal failure (CRF) not only causes an increase in oxidative stress in the body, but also leads to a decreased clearance of advanced glycation end products. CRF-related arteriosclerosis can also be interpreted by the five-phase engendering and restraining theory of pathology in traditional Chinese medicine (TCM). According to five-phase theory, water restrains fire, that is, diseases of the kidney are transmitted to the heart. Moreover, because the heart governs the blood vessels, diseases of the kidney indirectly damage the blood vessels. Therefore, from the principles of treatment and nursing care in TCM, arteriosclerosis treatment and care can be used according to the formation mechanism. Furthermore, treatment that attenuates renal dysfunction progression may improve kidney function and achieve the aim of preventing or reducing the risk of developing arteriosclerosis.

Keywords: Chronic Renal Failure; Advanced Glycation end Products; Arteriosclerosis; Hypertension; Traditional Chinese Medicine

Abbreviations: AGEs: Advanced Glycation End Products; CRF: Chronic Renal Failure; TCM: Traditional
Chinese Medicine; CML: Carboxymethyl-lysine; DG: Deoxyglucosone. 


\section{Nursing \& Healthcare International Journal}

\section{Chronic Renal Failure and Hypertension}

Hypertension has continually posed a serious threat to human health and life. In generally, hypertension can be categorized into primary hypertension of unknown cause or complicated hypertension with CRF or secondary hypertension diabetes associated with diabetes. Previous studies have suggested that the mechanisms of secondary hypertension due to CRF are as follows Figure 1:

- Sodium and water retention: Increased blood volume in repose to a decline of glomerular filtration rate due to severe CRF will result in an increase in cardiac output and blood pressure.

- Renin-angiotensin-aldosterone system activation: Kidney disease-associated renal vascular disease leads to a decrease in renal blood flow, which increases renin secretion and activates vasoconstrictors. The constriction of the resistant arterioles then leads to hypertension. In addition, angiotensin can also stimulate the secretion of aldosterone, increasing sodium and water reabsorption from renal tubules, and increasing sodium and water retention.

- Renal sympathetic nerves: Norepinephrine released from nerve endings during activation of the renal sympathetic nerves stimulates glomerular cells, which increases renin secretion, and promotes renal tubular reabsorption of sodium and water.

- Decreased in hypotensive substances of the kidney: Renal medullary interstitial cells papilla and renal cortical glomeruli cells induce dilation of blood vessels, inhibit renin, and excrete prostaglandin E2 and I2 with water. When renal tissue damage occurs, the quantity of secreted antihypertensive substances is reduced, which indirectly affects blood pressure.

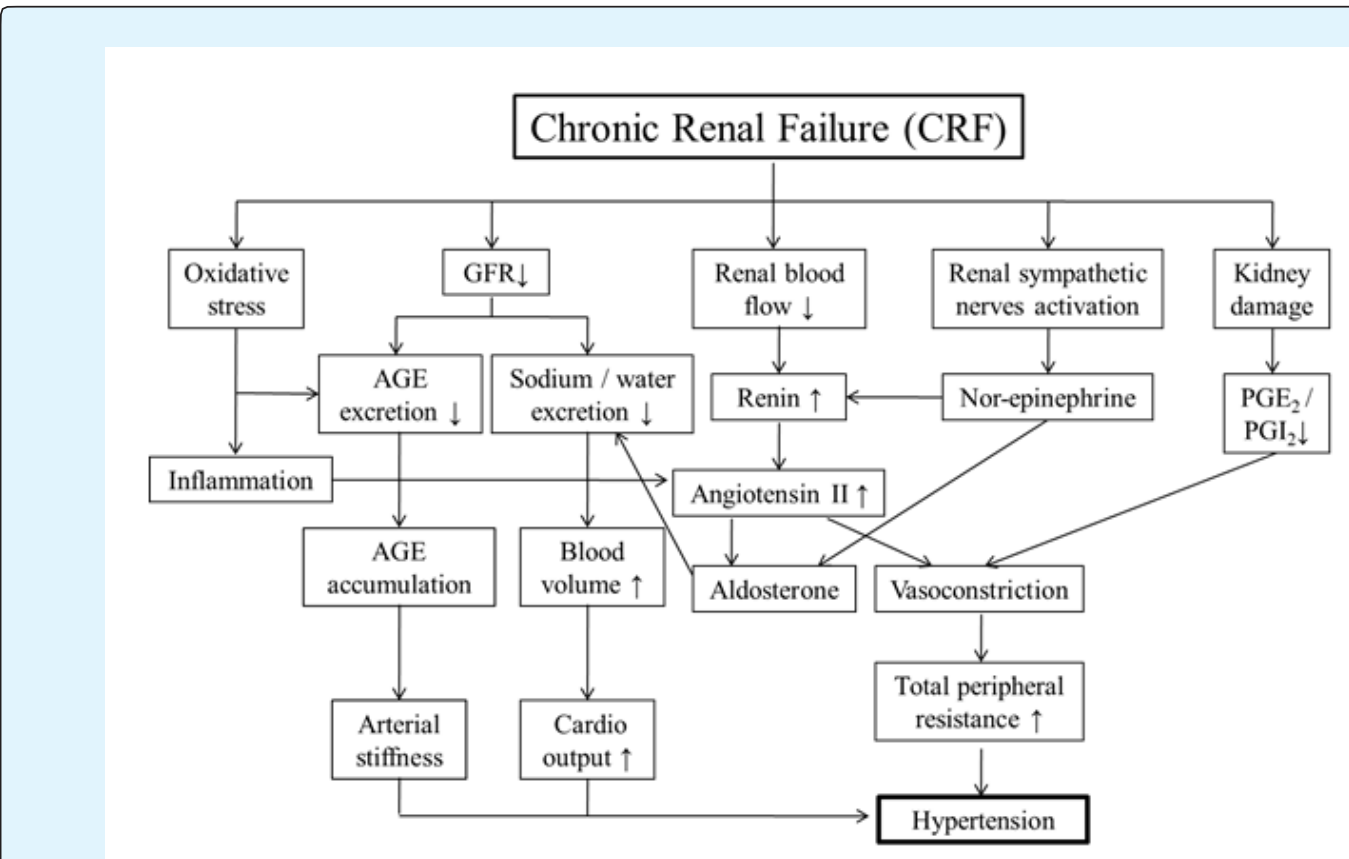

Figure 1: The mechanism of secondary hypertension due to chronic renal failure.

\section{Mechanism of Chronic Kidney Disease- Related Atherosclerosis}

In addition to the above mechanisms, newer studies have found that the incidence of hypertension is related to deposition of advanced glycation end products (AGEs) on the arterial wall. The interaction between AGEs and arterial collagen will lead to arteriosclerosis [1].

\section{Formation of AGEs}

The reaction between proteins and sugars in vivo is known as glycosylation and can be classified into two categories according to the type: the first type is an enzyme involved in the catalytic reaction in the endoplasmic reticulum or Golgi apparatus, where the proteins undergo glycosylation. The other one is a nonenzymatic glycation reaction, such as the formation of 


\section{Nursing \& Healthcare International Journal}

AGEs; this type of reaction is also known as the Maillard reaction. The Maillard reaction comprises three phases: initial phase, proliferative phase, and terminal phase [2].

Early stage: The carbonyl group of a reducing sugar (e.g., glucose) reacts with the primary amino group of the proteins to produce an unstable Schiff base, which undergoes rearrangement to form more stable Amadori products. This phase is a reversible reaction, and does not require oxygen.

Intermediate stage: Amadori products further react with lysine in proteins, and produce $\mathrm{N}-\varepsilon$-carboxymethyl-lysine (CML) with the involvement of the heavy metal ions and oxygen in catalysis. The sugar moiety is dehydrated to form deoxyglucosone (DG).

Advanced stage: Pyrraline formed from the reaction between highly reactive dicarbonyl-deoxyglucose and the residual lysine in the protein or pentosidine formed from the reaction between pentose, residual lysine and arginine in the protein become irreversible AGEs.

Based on this, it can be understood that AGEs refer to highly reactive compounds; since their formation process does not require enzymes, the reaction time is long. Therefore, long-lived proteins (e.g., collagen) tend to form AGEs and crosslink, causing loss of protein elasticity. If the reaction takes place in arterial collagen, it can lead to arteriosclerosis [3].

\section{Metabolism of AGEs}

When the glucose concentration in the body is too high (e.g. hyperglycemia due to diabetes), heavy metal ions or oxidative stress are present in the body, the formation of AGEs accelerates and is an irreversible process. AGEs must undergo macrophage phagocytosis to be degraded into reversible low molecular weight-AGEs, and subsequently be excreted by the kidneys. Therefore, the concentration of AGEs in vivo is closely related to renal function; when renal function is normal, the clearance rate is $0.72 \mathrm{ml} / \mathrm{min}$. Due to the diminished capacity for renal clearance of AGEs in CRF patients, AGEs accumulated in the body and cause arteriosclerosis $[4,5]$.

\section{TCM Theory and Pathophysiologic Mechanism of CRF and Arteriosclerosis- Induced Hypertension}

According to TCM theory, hypertension belongs to the category of dizziness; the condition is often caused by inhibited emotions, dietary irregularities, or internal damage due to vacuity detriment, leading to kidney and liver yin vacuity, ascendant hyperactivity of liver yang, or yin-yang disharmony, inducing hypertension. The primary pathology is regarded as yin deficiency as the root and hyperactivity of yang as the tip; this extends to the heart, liver, and kidney; the kidney is the root, the liver is the tip, and the condition is shifted to the heart [6]. The primary pattern types according to TCM theory are as follows: yin deficiency with yang hyperactivity, phlegmdamp collecting internally, dual deficiency of yin and yang, liver-kidney yin deficiency, dual deficiency of qi and yin, qi stagnation with blood stasis, qi deficiency with blood stasis, etc. The basic pathogenesis of the condition ranges from internal fire, yang hyperactivity, blood stasis, or phlegm turbidity [7]. Among these, with the exception of blood stasis due to qi deficiency with poor blood movement, qi stagnation with inhibited flow of blood, or congealing cold-related stasis obstruction of blood vessels, the condition is also possible to be due to blood stasis obstruction created by a stagnant and rough pulse. Moreover, when blood is stagnant and pulse is rough according to TCM theory, it can be known that the disease may have originated from the kidneys. Based on the TCM five-phase engendering and restraining theory of pathology, it is mentioned in the Jade Swivel Treatise on True Visceral Pulses in Plain Questions (Su Wen) that "The kidneys receive qi from the liver and transmit it to the heart." Based on this, it can be seen that the kidney diseases are conveyed to the heart, and because the heart also governs the blood vessels, TCM theory also regards that kidney diseases affect the function of the vessels. This point is in agreement with Pathophysiologic mechanism that CRF-induced arteriosclerosis leads to hypertension [8].

Of the causes of dizziness, emotions, diet, internal damage, etc. can cause increased endogenous oxidative stress, damaging the body and accelerating the formation of AGEs and inducing arteriosclerosis [9]. For example, of the seven emotions in TCM theory, fear damages the kidney, and the kidney conveys this to the heart. From the viewpoint of Pathophysiologic mechanism, internal injuries cause immune cells to function in addition to emotional abnormalities, resulting in increased endogenous oxidative stress and kidney disease. Kidney disease leads to the production of more oxidative stress, and an increase in the formation of AGEs [10]. At the same time, kidney disease causes a decline in kidney function, affecting the excretion of AGEs, which accumulate in the blood vessels, eventually leading to arteriosclerosis and hypertension. In addition, a reduction in renal blood flow caused by arteriosclerosis will initiate the reninangiotensin-aldosterone system and exacerbate hypertension. Therefore, to determine the treatment 


\section{Nursing \& Healthcare International Journal}

principles based on these viewpoints, tonify the kidney and blood-activating are the two main focuses. The purpose of tonify the kidney is to improve renal function in order to maintain normal AGEs excretion, whereas the purpose of blood-activating is to promote blood circulation, and subsequently reduce the formation or accumulation of AGEs in the blood vessels, which could delay the onset of arteriosclerosis.

\section{Treatment and Nursing Care Through Tonify the Kidney and Blood-Activating According to TCM Theory}

The basic pathology of CRF in TCM is debilitation and vanquishing of the spleen and kidney, which causes internal stagnation of water-dampness and internal static blood obstruction; the course of the disease starts with excess and moves to deficiency [11], therefore the course of treatment should be to treat both the root and tip by reinforce the healthy qi and eliminate the pathogenic factors. Treatment of the root focuses on tonify and replenish the spleen and kidney to maintain remaining kidney function [12], whereas treatment of the tip focuses on draining turbidity and clearing heat to induce diuresis and alleviate edema. Reduction of sodium and water retention are performed in order to avoid hypertension caused by the increased blood volume, and activate blood and resolve stasis is also performed throughout the course of treatment; This not only ameliorates renal blood stasis and increases renal blood supply in order to facilitate the recovery of kidney damage, but also slow the development of arteriosclerosis to ameliorate hypertension [13].

\section{TCM Treatment}

Many previous studies have shown that treatment methods to tonify the kidney and activate the blood are effective in improving renal function. For example, when medicinal such as Liu-wei Di-huang pill, which includes the constituents of cooked rehmannia root (shu di huang), cornus (shan zhu yu), root cortex of paeonia (Mu dan pi), alisma orientalis (ze xie), poria cocos (fu ling), dioscorea Alata (yam), are used to treat CRF, blood urea nitrogen and serum creatinine can be effectively reduced, increasing endogenous creatinine clearance, while effectively reducing triglyceride and cholesterol levels $[14,15]$. Pharmacological research has shown that these medicinals contain many biologically active ingredients. They can not only improve renal function and antioxidant capacity, but also inhibit the formation of AGEs [16-20].

\section{TCM Nursing Care}

In addition to pharmacotherapy, correct determination of pattern to elucidate the pathogenesis of the condition can effectively improve kidney function in patients with CRF and reduce oxidative stress. Common clinical care measures are as follows:

Emotional care: Emotions indicate the changes in psychophysiological activities that can directly affect the function of organs. According to TCM theory, inhibition of emotions is an internal cause of diseases. In the Great Treatise on the Correspondences and Manifestations of Yin and Yang in Su Wen of the Huangdi Neijing, states that "anger damages the liver, joy damages the heart, thought damages the spleen, sadness damages the lungs, and fear damages the kidneys." This explains that when there is a disturbance in emotions, it can lead to disorders of qi, blood, yin, and yang, thereby causing dysfunction of the organs [21]. The results of past pathophysiologic studies have shown that mental stress can cause increased endogenous oxidative stress, resulting in arteriosclerosisinduced hypertension [22]. Therefore, for patients with different conditions, the appropriate method of emotional care for patients should be selected to enlighten patients, dispel doubts, or use diversionary techniques to achieve a resolution of emotional stagnation. When necessary, restrictive or suggestive methods can be used to relieve patient anxiety and tension related to negative emotions, ameliorating the progression of the disease [21].

Diet therapy: The effects of diet are extremely important for patients with CRF. In addition to avoiding a highsodium, high-fat diet, it is also important to reduce the intake of foods that promote the development of AGEs, such as foods high in sugar, barbecued foods [23], establish good eating habits, and avoid improper diet that leads to spleen deficiency, which increased endogenous oxidative stress [24], therefore reduce the risk of onset of arteriosclerosis. Furthermore, according to the TCM theory of "same origin of Chinese medicines and foods", foods have the four qi and five flavors, therefore when performing diet therapy, it is advisable to follow the principle of "salt damages the kidney, and bland flavors percolate dampness", that is, patients should follow a lowsodium, bland diet and increase the intake of foods that tonify the kidney and replenish qi, such as black beans, black rice, black fungus, dates, flowery knotweed, silky fowl, and sea cucumber to improve renal function. Modern medical research has revealed that these foods also have a strong antioxidant capacity [25]. 


\section{Nursing \& Healthcare International Journal}

Daily life care: The living environment and living conditions for the health of the body also have a great impact. The living environment should be kept well ventilated and be located in a shady area; By avoiding overexposure to ultraviolet radiation, air pollution and smoking to reduce exogenous oxidative stress, it is possible to reduce the formation of AGEs and reduce the incidence of arteriosclerosis [26]. Irregular lifestyle and staying up late are likely to cause increased endogenous oxidative stress, resulting in damage to internal organs. Therefore health guidance must focus on educating the patient to maintain a regular lifestyle. As it is written in the Treatise of Heavenly Truth from Remote Antiquity in in Su Wen of the Huangdi Neijing, "their eating and drinking is moderate, rising and resting had regularity, they do not tax themselves with meaningless work. Hence, they are able to keep their physical appearance and spirit together, and to exhaust the years allotted by heaven. Their life span exceeded one hundred years before they depart from this life." In addition to these points, sexual activity should be limited. According to TCM theory, kidney is the root of earlier heaven and the organs are the storehouses of essence; when kidney essence is sufficient, the bowels and viscera are strong. For this reason, CRF patients should limit sexual activity in order to maintain adequate kidney essence and avoid further exacerbation of the condition.

\section{Conclusion}

In CRF patients, the activation of the reninangiotensin-aldosterone system causes arteries to constrict and increases blood volume, leading to hypertension, the increase in endogenous oxidative stress induced by kidney disease accelerates the formation of AGEs. At the same time, renal failure causes insufficient renal function, excretion of AGEs decreases. When both are present, excessive accumulation of AGEs occurs on the blood vessel walls, leading to the development of arteriosclerosis, which further exacerbates hypertension [27]. Therefore, in considering the methods to prevent the disease, it is necessary to consider the TCM theory of "treating disease before it arises" and the pathogenic mechanisms of the condition as elucidated by pathophysiology to combine treatment using TCM and pathophysiology in order to reduce CRF complicated by arteriosclerosis, which can lead to hypertension. The principles of treatment are based on the five-phase engendering and restraining theory of pathology in TCM. That is, because the kidney invariably transmits the disease to the heart, concomitant treatment of the root and tip is needed to boost qi and nourish the blood, drain dampness and down bearing the turbid, activate blood and resolve stasis, thereby slowing the progression of CRF and ameliorating renal function. Moreover, by combining the emotional care, diet therapy and daily life care of TCM, it is possible to reduce oxidative stress to prevent excessive formation of AGEs, while maintaining the normal excretory mechanism of AGEs, maintaining constant blood volume, and preventing the constriction of shrinkage-resistant arteries in order to achieve the common goal to reduce CRF-induced hypertension.

Conflicts of Interest: None declared.

\section{References}

1. McNulty M, Mahmud A, Feely J (2007) Advanced glycation end-products and arterial stiffness in hypertension. Am J Hypertens 20(3): 242-247.

2. Singh R, Barden A, Mori T, Beilin L (2001) Advanced glycation end-products: a review. Diabetologia 44(2): 129-146.

3. Ikeda K, Higashi T, Sano H, Jinnouchi Y, Yoshida M, et al. (1996) N (epsilon)-(carboxymethyl) lysine protein adduct is a major immunological epitope in proteins modified with advanced glycation end products of the Maillard reaction. Biochemistry 35(24): 8075-8083.

4. Araki N, Higashi T, Mori T, Shibayama R, Kawabe Y, et al. (1995) Macrophage scavenger receptor mediates the endocytic uptake and degradation of advanced glycation end products of the Maillard reaction. Eur J Biochem 230(2): 408-415.

5. Miyata T, Wada Y, Cai Z, Iida Y, Horie K, et al. (1997) Implication of an increased oxidative stress in the formation of advanced glycation end products in patients with end-stage renal failure. Kidney Int 51(4): 1170-1181.

6. Wang J, Xiong X (2013) Evidence-based chinese medicine for hypertension. Evid Based Complement Alternat Med 2013: 978398.

7. Wang J, Xiong X, Liu W (2014) Traditional chinese medicine syndromes for essential hypertension: a literature analysis of 13,272 patients. Evid Based Complement Alternat Med 2014: 418206. 


\section{Nursing \& Healthcare International Journal}

8. Chuang YC, Wu MS, Su YK, Fang KM (2012) Effects of olmesartan on arterial stiffness in rats with chronic renal failure. Cardiovasc Diabetol 11: 66.

9. Kizer JR, Benkeser D, Arnold AM, Ix JH, Mukamal KJ, et al. (2014) Advanced glycation/glycoxidation endproduct carboxymethyl-lysine and incidence of coronary heart disease and stroke in older adults. Atherosclerosis 235(1): 116-121.

10. Nishizawa Y, Koyama H, Inaba M (2012) AGEs and cardiovascular diseases in patients with end-stage renal diseases. Journal of renal nutrition 22(1): 128133.

11. Yang CC (2010) Progress on TCM Treatment of Chronic Renal Failure (CRF). Taipei Journal of Chinese Medicine 16(4): 76-87.

12. Chen WD (2016) Treatment of edema: A case report. Journal of Traditional Chinese Internal Medicine 14(1): 23-32.

13. Kou MC (2009) Treatment of Hypertension. Taipei Journal of Chinese Medicine 15(3): 40-44.

14. Lin CC (2008) Integrated TCM treatment on chronic kidney function insufficiency: A case report. Taiwan Journal of Clinical Chinese Medicine 14(1): 45-52.

15. Xu ZJ, Shu S, Li ZJ, Liu YM, Zhang RY, et al. (2017) Liuwei Dihuang pill treats diabetic nephropathy in rats by inhibiting of TGF-beta/SMADS, MAPK, and NF$\mathrm{kB}$ and upregulating expression of cytoglobin in renal tissues. Medicine 96(3): e5879.

16. Zhu H, Wang Y, Liu Z, Wang J, Wan D, et al. (2016) Antidiabetic and antioxidant effects of catalpol extracted from Rehmannia glutinosa (Di Huang) on rat diabetes induced by streptozotocin and high-fat, high-sugar feed. Chinese medicine 11: 25.

17. Baek GH, Jang YS, Jeong SI, Cha J, Joo M, et al. (2012) Rehmannia glutinosa suppresses inflammatory responses elicited by advanced glycation end products. Inflammation 35(4): 1232-1241.

18. Xu H, Shen J, Liu H, Shi Y, Li L, et al. (2006) Morroniside and loganin extracted from Cornus officinalis have protective effects on rat mesangial cell proliferation exposed to advanced glycation end products by preventing oxidative stress. Can J Physiol Pharmacol 84(12): 1267-1273.
19. Ha do T, Ngoc TM, Lee I, Lee YM, Kim JS, et al. (2009) Inhibitors of aldose reductase and formation of advanced glycation end-products in moutan cortex (Paeonia suffruticosa). J Nat Prod 72(8): 1465-1470.

20. Szeto YT, Lei PC, Ngai KL, Yiu AT, Chan CS, et al. (2009) An in vitro study of the antioxidant activities and effect on human DNA of the Chinese herbal decoction 'Liu Wei Di Huang'. Int J Food Sci Nutr 60(8): 662-667.

21. Zhang YM, Xia LC (2009) The Analysis of TCM's Emotional Nursing. Liaoning Journal of Traditional Chinese Medicine 36(6): 1019-1020.

22. Inoue $N$ (2014) Stress and atherosclerotic cardiovascular disease. J Atheroscler Thromb 21(5): 391-401.

23. Chao PC, Hsu CC, Yin MC (2009) Analysis of glycative products in sauces and sauce-treated foods. Food Chemistry 113(1): 262-266.

24. Wang CX, Cui JP, Lu AP, Liu CX, Qin JS (2007) Study on the Change about of Telomere in Liver Tissue of Deficiency in Spleen Model Rats and the Relationship between This Change and Oxidative Stress. Chinese Archives of Traditonal Chinese Medicine 25(12): 2448-2450.

25. Mai Q, Li X, Wu Y, Zhou C (2010) BP model for relation between anti-aging and four natures, five flavors and meridian tropism of Chinese prescription medicine, Zhongguo Zhong Yao Za Zhi 35(24): 33713376.

26. Pulliero A, Godschalk R, Andreassi MG, Curfs D, Van Schooten FJ, et al. (2015) Environmental carcinogens and mutational pathways in atherosclerosis. Int J Hyg Environ Health 218(3): 293-312.

27. Rabbani N, Sebekova K, Sebekova K, Heidland A, Thornalley PJ (2007) Accumulation of free adduct glycation, oxidation, and nitration products follows acute loss of renal function. Kidney Int 72(9): 11131121. 